\title{
Análisis de la información y documentación científica española sobre el fenómeno de las smart cities, el hábitat de los nativos digitales
}

\author{
Francisco Cabezuelo-Lorenzo \\ Fernando Bonete-Vizcaíno \\ María Sánchez-Martínez
}

\section{Resumen}

Estudiosos de todo el mundo se están centrando en el estudio del fenómeno de las ciudades inteligentes. La producción bibliográfica española sobre este tema ha crecido exponencialmente en los últimos años. Las nuevas ciudades inteligentes se fundamentan en nuevas visiones de desarrollo urbano que integran múltiples soluciones tecnológicas ligadas al mundo de la información y de la comunicación, todas ellas actuales y al servicio de las necesidades de la ciudad. La literatura en español sobre este tema proviene de campos tan diferentes como la Arquitectura, la Ingeniería, las Ciencias Políticas y el Derecho o las Ciencias Empresariales. La finalidad de las ciudades inteligentes es la mejora de la vida de sus ciudadanos a través de la implementación de tecnologías de la información y de la comunicación que resuelvan las necesidades de sus habitantes, por lo que los investigadores del campo de las Ciencias de la Comunicación y de la Información tienen mucho que decir. Este trabajo analiza un total de 120 textos y concluye que el fenómeno de las ciudades inteligentes será uno de los ejes centrales de la investigación multidisciplinar en los próximos años en nuestro país.

\section{Palabras clave}

Comunicación Digital; Ciudades Inteligentes; Internet; Big data; Producción científica española

\section{Analysis of the Spanish Information and Scientific Literature about the Smart-City Phenomenon, the Digital Native's Habitat}




\section{Abstract}

Academics from all over the world are focused on the phenomenon of smart city. Spanish literature on the topic has grown in the last years. Smart cities are based in new urban development visions that integrate multiple information and communication technology solutions in a secure fashion to manage all the city assets. Spanish literature about this topic comes from different fields as Architecture, Engineering, Political Science, Business and Law. The goal of building a smart city is to improve quality of life by using ICT technology to improve the efficiency of services and meet residents' needs, so the researchers from the field of Communication and Information Sciences have a lot to say about it. This study analyses 120 units and consider that the smart city phenomenon is a reality that will be on the center of a challenging new multifunctional research.

\section{Keywords}

Digital Communication; Smart Cities; Internet; Big data; Spanish Scientific Literature Spain.

\section{Justificación e introducción}

En la actualidad, tanto en España como en el extranjero, conviven diferentes corrientes teóricas que explican la historia y evolución del nacimiento y desarrollo del nuevo fenómeno de las "ciudades inteligentes" (traducción aceptada en lengua española del término original inglés smart cities). Así, existe un grupo de académicos y de profesionales que defiende el origen estadounidense del fenómeno y que usan la expresión anglosajona para hablar del fenómeno, tanto en su dimensión internacional como en su implantación en España. Esta corriente mantiene el uso del término en lengua inglesa para hacer referencia a un fenómeno que ha evolucionado y ampliado sus campos de actuación, saltando del Urbanismo y la Arquitectura a las Ciencias de la Información y de la Comunicación, pero no ha variado su denominación.

Sin embargo, existen otros sectores otras posiciones que, por diferentes motivos, difieren de esta postura tanto en los orígenes como en la evolución. En esta corriente se encuentran diversos autores que apoyan la idea de que la puesta en marcha de actividades y acciones de servicio a través de medios digitales y del análisis de big data ya se daba en España antes de la llegada, moda y/o implantación de los conceptos y tendencias internacionales. 
El mundo de las ciudades inteligentes tiene además una honda relación con el mundo del tratamiento de datos y las Ciencias de la Información y de la Documentación. La mayoría de las iniciativas tecnológicas en materia de smart cities se fundamentan en el desarrollo de aplicaciones de que analizan y generan nuevos datos útiles para la ciudadanía, fruto del análisis de los ahora tan en boga big data o "volúmenes masivos de información tanto estructurada como no estructurada que es dinámica por naturaleza que es recogida durante cierto periodo de tiempo y que requiere de métodos computacionales para extraer conocimiento de ella" (Verbeke et al., 2014:02). Se trata de grandes bloques de datos que vienen definidos por tres elementos claves como son su tamaño, momento de producción y forma de recolección.

Paradójicamente, a pesar de la importancia económica y tecnológica que la historia y la evolución del fenómeno de las ciudades inteligentes pueda tener para el ejercicio profesional de las nuevas generaciones de titulados universitarios, hasta la fecha no se han puesto en marcha estudios que hayan revisado y comparado lo escrito hasta el momento sobre esta nueva parcela del conocimiento. De la misma manera, a modo de justificación de este estudio, es preciso afirmar que Del mismo modo ha llegado la hora de generar documentos de trabajo que sirvan como base para abordar este objeto de estudio desde una sólida perspectiva que genere pilares de referencia para su posterior desarrollo académico. El fenómeno de las smart cities es objeto de investigación creciente. Va más allá de una moda. Es toda una realidad. En este contexto resulta interesante compilar la producción científica referente al ámbito de estudio para obtener el panorama general de las distintas posiciones que tanto los profesionales como los académicos tienen sobre la historia y evolución en España del fenómeno.

\section{Contexto y marco teórico}

Uno de los títulos clásicos del cine mudo, la magistral película Metrópolis (dirigida por Fritz Lang y estrenada en 1927) se adelantaba a una ciudad-estado de tipo futurista (supuestamente ambientada en el año 2026) en la que se imaginaba un mundo en el siglo XXI con rascacielos de siluetas similares a los de Manhattan y en los que sus 
habitantes eran gente tremendamente infeliz. En la cinta se muestra una humanidad en la que los individuos viven aprisionados entre frías y gigantescas paredes de hormigón, con la libertad y la creatividad anuladas, en un ambiente asfixiante, sombrío y explotador. En la cinta, las clases sociales menos pudientes vivían escondidas en el subsuelo, donde trabajaban a destajo y de donde no podían salir bajo ningún concepto, mientras que por otro lado los empresarios y sus pensadores protectores eran los únicos habilitados para pasear prepotentes y alegres por la superficie exterior. Aunque las noticias de sucesos demuestran casos puntuales de empleados explotados encerrados en talleres ilegales, la verdad es que no es la norma general, así que si el cineasta austriaco Fritz Lang (1890-1976) pudiera levantar hoy la cabeza y mirar a su alrededor, aunque no estaba equivocado del todo en algunos aspectos, la verdad es que no tendría otra opción que reconocer su equívoco (Llobell, 2012).

\subsection{El desarrollo de las ciudades}

Los informes de los organismos nacionales corroboran como los habitantes del planeta siguen mudándose a las ciudades porque, a pesar de los inconvenientes de las grandes urbes, las aglomeraciones urbanas son el principal polo de dinamismo económico y social. Del mismo modo, son el principal laboratorio de creatividad y descentralización de la humanidad. Lo urbano no es ese infierno de desdichados que se imaginaba. El siglo XXI es sin duda alguna el siglo de las ciudades, según Llobell (2012). Por eso, es preciso tener en consideración esta realidad y estudiarla desde múltiples perspectivas, incluidas las Ciencias de la Información, Documentación y Comunicación. Las ciudades pueden ser entornos formidables pero también terribles ollas a presión, sobre todo en momentos de crisis, como ha ocurrido en los últimos años, donde la crisis se ha sentido sobre todo en la industria de la información y de la comunicación (Cabezuelo, 2013: 703).

De acuerdo con los datos de la Organización de Naciones Unidas (ONU), recogidos por Pascual (2012), cada mes más de cinco millones de nuevos ciudadanos se adentran en su asfalto en busca de una vida mejor. Según la Conferencia de las Naciones Unidas sobre el Desarrollo Sostenible, celebrada en Río de Janeiro en 2012, la mitad de la humanidad vive en ciudades. Si en 1900 solo el 13 por ciento de la población vivía en las grandes 
metrópolis, en 2050 se prevé que ese número aumente hasta un 70 por ciento.

La población urbana ha aumentado desde los 750 millones de personas -que aproximadamente la constituían en 1950-, hasta los 3.600 millones en 2011. Se estima que hacia 2030 casi un 60 por ciento de la población mundial residirá en zonas urbanas. En España lo hace ya más del 80 por ciento, según datos del Atlas Estadístico de las Áreas Urbanas de España del Ministerio de Fomento. Es decir, dentro de pocas décadas habrá más de 6.000 millones de urbanitas en el planeta. Si no las volvemos más inteligentes y sostenibles, las ciudades reventarán.

\subsection{El desarrollo de las tecnologías}

En este contexto entran en juego las Tecnologías de la Información y de la Comunicación (en adelante TIC). Los avances tecnológicos que se han producido en el último cuarto de siglo, y principalmente el desarrollo de Internet, han propiciado el acceso de los ciudadanos a una cantidad infinita y variada de contenidos digitales desarrollados no solo a través de iniciativas privadas sino también públicas. El último factor es el crecimiento de los medios digitales y la expansión del entorno digital.

Redes sociales, plataformas digitales, dispositivos móviles, tabletas, la mensajería instantánea o los periódicos digitales han creado un nuevo ecosistema de relaciones informativas, políticas y ciudadanas, según Manuel Castells (2008: 13-14). Una de las consecuencias de la digitalización, ya sea texto, audio, video o imágenes bien a través de la transformación de contenido analógico en un conjunto de dígitos binarios o directamente por la captura digital de la información, ha motivado que los contenidos puedan ser vistos y consumidos a través de diferentes dispositivos digitales propiciando una convergencia tecnológica y multiplataforma (Sánchez-Martínez e Ibar Alonso, 2015: 87). Internet se ha convertido hoy en la principal herramienta para la búsqueda de información desde ámbitos universitarios, y no solo estudiantiles, como ya investigó Caldevilla Domínguez (2010: 141) a la hora de encumbrar Internet casi como fuente única de información para el alumnado universitario. Del mismo modo, en la industria de la comunicación, Internet ha colocado a los medios en una posición difícil de entender 
para editores, redactores, documentalistas, gestores y también para los usuarios/ lectores que tienen una nueva percepción de la realidad informativa (Marcos Recio, Sánchez Vigil \& Serrada Gutiérrez, 2009: 43).

Además, la aparición de dispositivos portátiles como las tabletas y los smartphones han hecho florecer un universo de aplicaciones que ayudan a los usuarios en su vida cotidiana a acceder a una amplia tipología de contenidos como son el ocio, la cultura, el turismo, la salud, la educación o el comercio electrónico. Evidentemente no todas las aplicaciones que se desarrollan son útiles o interesantes y en ocasiones no son más que una prolongación adaptada de los contenidos de las tradicionales páginas webs corporativas de empresas e instituciones, entre las que se encuentran los ayuntamientos.

Las ciudades, entendidas como municipios o entidades locales, independientemente de su tamaño y población, se han constituido como los principales centros de desarrollo económico, social y cultural, en las actuales sociedades contemporáneas; el entorno propicio para el desarrollo de la creatividad y la innovación, particularmente la innovación social. Todos estos desarrollos son fundamentales a la hora de alcanzar la gestión inteligente de las ciudades y deben estar en un lugar preferente en los calendarios, acciones de empresas y administraciones públicas. Las empresas y consistorios involucrados en el proceso de creación y adaptación de las ciudades están implantando infraestructuras y dispositivos dirigidos a un amplio marco de acción de vasta y muy variada influencia que va desde la eficiencia energética, gasto urbano, movilidad, la reducción del tráfico y la contaminación o la mayor agilidad ante la administración, entre otros.

Desde el momento en el que los ciudadanos comienzan a acceder a la red, las descargas, las visualizaciones y la navegación en los contenidos van conformando un tejido digital que permite conocer las necesidades, usos y gustos de estos usuarios. Dentro de los cambios y avances que se están produciendo, las ciudades se han convertido en un sistema en tiempo real que genera grandes cantidades de datos. Su uso inteligente, de la mano de las tecnologías de la información y comunicación, pueden facilitar el aprovechamiento de todo el potencial que las ciudades inteligentes encierran, no solo en 
lo que a eficiencia y sostenibilidad ambiental se refiere sino también en la estimulación de la innovación para la vida cotidiana en todos sus aspectos y con la creación de aplicaciones que permitan su difusión, accesibilidad, modelos de negocio nuevos y, por supuesto, que permitan avanzar tanto a las ciudades como a los ciudadanos.

Las infraestructuras basadas en la tecnología están contribuyendo a que las ciudades sean más eficaces en cuanto a la optimización de las necesidades de los ciudadanos, además de contribuir a su desarrollo socioeconómico. La nueva economía, por tanto, se hace un hueco también en las ciudades a través de las smart cities con soluciones innovadoras que a su vez consiguen una mayor calidad de vida para sus ciudadanos.

En el contexto de competitividad y de innovación de las ciudades inteligentes es necesaria la existencia de avances específicamente dirigidos a los ciudadanos a la hora de fomentar su integración en el ecosistema digital de las smart cities con la finalidad de permitirles el aprovechamiento de las TIC dentro de las ciudades inteligentes. Además, es necesario encontrar un valor añadido a través de la participación y la innovación, como parece ser objetivo clave del Programa Marco de Investigación e Innovación de la Unión Europea Horizonte 2020 (MECD y CDTI: 2014) y esto es posible en el contexto de cambio propiciado por las smart cities y sus tres motores (M2M, Internet of the Things (Internet de las cosas) y big data) que brindan una oportunidad única e inmejorable para fomentar el acceso, la difusión y promoción del turismo, el patrimonio cultural, la educación, el comercio o la salud a través de medios digitales electrónicos.

Sin embargo parece que no es tan fácil generar estrategias y modelos digitales de actuación en este sentido y dentro del contexto de las smart cities, en primer lugar por todas las áreas que abarca y en segundo lugar por la gestión de intangibles que representa en algunos casos como en el patrimonio, la educación o la cultura digital. Pero es necesario que, a través de estos nuevos impulsos y mejoras que sirven para generar innovación y riqueza en base a lo que las nuevas tecnologías permiten, se pueda contribuir a la construcción de ciudades en donde su identidad, patrimonio y los bienes y servicios que la componen puedan ser transmitidos, gestionados y disfrutados tanto por los ciudadanos que la integran como por los que la visitan. 


\title{
3. Objetivos y metodología
}

El objeto de estudio de este trabajo es el tratamiento académico y editorial dado por las diferentes revistas y publicaciones especializadas al fenómeno de las smart cities o ciudades inteligentes. Para empezar, es preciso señalar que no parece existir un consenso respecto al concepto de ciudad inteligente o de los elementos mínimos que han de estar presentes en una ciudad para que pueda ser considerada como tal. En España, y tras el análisis de toda la producción bibliográfica sobre la materia se puede afirmar que hay un consenso, pero no una unanimidad para una única definición conjunta o compartida. La acepción más extendida es la recogida en el Plan Nacional de Ciudades Inteligentes, que sigue la definición propuesta por el Grupo Técnico de Normalización 178 de AENOR (AEN/CTN 178/SC2/GT1 N 003) que afirma lo siguiente:

\begin{abstract}
"Ciudad inteligente (Smart City) es la visión holística de una ciudad que aplica las Tecnologías de la Información y de la Comunicación (TIC) para la mejora de la calidad de vida y la accesibilidad de sus habitantes y asegura un desarrollo sostenible económico, social y ambiental en mejora permanente. Una ciudad inteligente permite a los ciudadanos interactuar con ella de forma multidisciplinar y se adapta en tiempo real a sus necesidades, de forma eficiente en calidad y costes, ofreciendo datos abiertos, soluciones y servicios orientados a los ciudadanos como personas, para resolver los efectos del crecimiento de las ciudades, en ámbitos públicos y privados, a través de la integración innovadora de infraestructuras con sistemas de gestión inteligente."
\end{abstract}

Por tanto, sí puede convenirse que la aplicación de las TIC para mejorar la calidad de vida de sus habitantes y asegurar un desarrollo económico, social y ambiental sostenible es uno de los elementos comunes a todas las definiciones. Esta definición comúnmente aceptada nos lleva a pensar en las consecuencias de la misma. El fenómeno de las smart cities exige una nueva relación con ciudadanos, turistas, proveedores y trabajadores públicos basada en la transparencia, la rendición de cuentas, el adecuado uso y consumo de los recursos y la identificación temprana de necesidades. De hecho, el papel de las entidades locales como garantes de la correcta prestación de servicios públicos se ha vuelto más compleja en los últimos años por diversos motivos; entre otros, el mantenimiento de la Ley Reguladora de las Bases de Régimen Local del año 1985, que 
no permitía contemplar y abordar de forma sistemática la prestación de los servicios públicos en los distintos tipos de municipios. Con la aprobación de la Ley de Racionalización y Sostenibilidad de la Administración Local, de diciembre de 2013, se han acometido modificaciones en la prestación de los servicios básicos y en la determinación de los nuevos, de forma que se eviten duplicidades y solapamientos, facilitando una prestación más racional y con suficiente financiación.

Por otro lado, el objetivo general de esta investigación es analizar el origen y la evolución que la literatura sobre smart cities y el correspondiente fenómeno han tenido en España, en lengua española en la esfera hispanoamericana o en otras lenguas cooficiales del Estado Español. Рara ello, como objetivos específicos, se han planteado varios. El primero es conocer los diferentes planteamientos realizados por los académicos para explicar la historia y la evolución de las smart cities en España y, más específicamente:

- Delimitar y describir la evolución del concepto en España en los últimos 25 años, pero sobre todo desde lo que llevamos del siglo XXI, es decir, 2000-2015.

- Reconocer y contrastar las características y la evolución de la investigación en la materia en nuestro país.

- Intentar detectar las posibles concomitancias y puntos de inflexión que existen entre las diferentes corrientes de los profesionales y académicos de nuestro entorno.

Con el fin de alcanzar los objetivos planteados se seleccionó una muestra de artículos que nos permitiera afrontar una revisión crítica para conocer los planteamientos de los académicos españoles en cuanto a la historia y la evolución del fenómeno de las ciudades inteligentes en lengua española. Con este planteamiento se realizó una revisión bibliográfica de los textos publicados en España e Hispanoamérica relacionados con los orígenes y evolución de las smart cities. Para seleccionar la muestra se examinaron las revistas científicas contenidas en las prestigiosas bases de datos Dialnet en su versión 
Dialnet Plus (Universidad de La Rioja y universidades asociadas al proyecto). Estas mismas búsquedas se han corroborado también a través de las base de datos ISOC de Ciencias Sociales y Humanidades del Consejo Superior de Investigaciones Científicas (CSIC). Del mismo modo, este trabajo se realizó en paralelo a una investigación sobre la producción bibliográfica en lengua inglesa sobre las smart cities, para las que se usaron las bases de datos de Ebsco y Proquest. Finalmente, se decidió acotar el objeto de estudio a la producción en castellano y lenguas cooficiales autonómicas, para conocer cuál era el estado de la cuestión en la academia española.

Todas las búsquedas se planificaron utilizando una doble estrategia. Así, en un primer momento, se hizo una localización de términos en el título y resumen de los documentos incluidos en las bases seleccionadas, a través de los términos tanto en plural como en singular de "smart city" en inglés y/o "ciudad inteligente" en castellano (y otras lenguas cooficiales de España).

Posteriormente, en una segunda fase, se buscó la delimitación de algunas características o filtros para los documentos que se muestran en estos repositorios. Así respecto a la primera estrategia, los términos buscados se pueden agrupar en tres categorías o técnicas. Por un lado estarían las búsquedas ligadas a delimitar los resultados relacionados realmente con el origen y sentido del objeto de estudio (las ciudades inteligentes). En segundo lugar, se pretendía delimitar aquellos relacionados con las Ciencias de la Información y de la Comunicación. Y, finalmente, estarían los relacionados con la delimitación geográfica propia hispanoblante, descartando la producción anglosajona, pero no la española que usa el término original en inglés sin traducir en textos españoles o en lenguas cooficiales de España. En cuanto a la segunda estrategia, consistente en delimitar algunas características de los documentos mostrados, se ha tenido en cuenta el tipo de documento (artículos de revistas, capítulos de libros, libros), la materia y la cobertura geográfica iberoamericana.

Las búsquedas se realizaron entre mayo y junio de 2015. Como resultado de esta búsqueda inicial se recuperaron 120 artículos. Una vez seleccionada la muestra preliminar de textos, se realizó una revisión exhaustiva de los artículos y se excluyeron 
tanto aquellos documentos que estaban repetidos como los que, tras su lectura, no se consideraron pertinentes para el estudio por no hacer alusión de manera directa a los objetivos de la investigación ni a los términos fijados. La selección de los textos se hizo teniendo en cuenta las delimitaciones citadas establecidas con anterioridad a la realización de la búsqueda.

No existen diferencias entre el porcentaje de artículos obtenidos en cada base de datos, ya que Dialnet se ha usado como base principal. En este repositorio se han encontrado el 100 por ciento de los textos analizados (120 unidades de análisis), pero hay que destacar que en la base de datos de ISOC-CSIC solo se han encontrado 14 textos, que también estaban en Dialnet, motivo por el que ISOC fue descartada para buscar nuevas producciones, ya que no aportaba más unidades de análisis que Dialnet. Eran repetidas. Las 14 de ISOC también aparecían en Dialnet, por lo que suponen un 11 por ciento del total. Así, cabe señalar que un 100 por ciento de los artículos de la muestra se han encontrado en una única base de datos mientras que un 11,66 por ciento de los textos se pueden localizar en las dos bases de datos utilizadas.

\section{Análisis y resultados}

Sin duda alguna, la primera valoración que merece el análisis de los resultados es la cuestión de la temporalidad o moda en el estudio del fenómeno de las ciudades inteligentes en nuestro país. Las 120 piezas de análisis (artículos y capítulos de libros) están firmadas por un total de 134 autores. Hay piezas individuales, pero también firmadas por dos y tres autores. El total de unidades de análisis cubre los últimos 25 años, destacando el hecho de que sólo una corresponde a la década de 1990-2000. Es la única del siglo pasado registrada en Dialnet. El trabajo pionero de la bibliografía española registrado a día de hoy en esta materia corresponde a M. Aymerich y Ch. Rabanal, autores del capítulo titulado “Ciudades Inteligentes” (págs. 137-151), publicado en el libro Tráfico y transporte en ciudades de escala intermedia, coordinado por Luis Álvarez Vázquez y José Ramón Navarro Vera en 1990, editado por el Ayuntamiento de Alicante/Ajuntament d'Alacant. 
No vuelven a registrase trabajos sobre ciudades inteligentes o smart cities, hasta el año 2002 cuando en Argentina, Roberto Zubieta y Tedy Woodley publican su trabajo "Proyecto argentino de ciudades y municipios inteligentes" en el número 90 de Revista AHCIET-Revista de Telecomunicaciones, una publicación de la Asociación Hispanoamericana de Centros de Investigación y Empresas de Telecomunicaciones (AHCIET).

En el año 2006 (un trabajo) vuelven a aparecer publicaciones sobre las ciudades inteligentes y desde entonces prácticamente no dejan de publicarse de manera creciente, pasando de los 2 de 2007 a los 4 en 2008 y 7 en 2011. En 2012 se localizan ya 27 unidades de análisis y otras 27 también en 2013. En el año 2014 la cifra se dispara hasta los 41 trabajos publicados. La previsión para este año es la de un crecimiento exponencial como nunca antes visto.

En lo que respecta al contenido y sus perspectivas de aproximación al tema, con el fin de alcanzar los objetivos expuestos, se ha realizado una revisión crítica de la literatura académica obtenida, tratando de detectar los puntos de inflexión que propician la existencia de varias posturas diferenciadas respecto al origen del fenómeno de las smart cities y su evolución.

Conceptos como "sostenibilidad", "movilidad", "TIC" o "destino inteligente" son tan solo algunos de los términos más usados a la hora de repasar la literatura existente sobre la materia. Las asociaciones profesionales y las entidades locales, al ir avanzando en este proceso inteligente de modernización, son los principales promotores de estas publicaciones. Conforme a Dialnet, las etiquetas más repetidas, además de smart city o ciudad inteligente tanto en singular como en plural, son "innovación", "tecnología", "living labs", "calidad de vida" o "colaboración público-privada", entre otros

A continuación se muestran dos tablas con las publicaciones periódicas españolas de referencia en el campo de las ciudades inteligentes y que han sido analizadas. Suponen un total de 58 revistas de tipo tanto académico como profesional. 


\begin{tabular}{|c|c|}
\hline \multicolumn{2}{|c|}{ Listado de publicaciones que albergan textos sobre smart cities } \\
\hline $\begin{array}{c}\text { AENOR: Revista de la normalización y la } \\
\text { certificación }\end{array}$ & Fórum Calidad \\
\hline $\begin{array}{l}\text { Afkar ideas: Revista trimestral para el } \\
\text { diálogo entre el Magreb, España y Europa }\end{array}$ & Hábitat y sociedad \\
\hline $\begin{array}{c}\text { Agenda de la empresa andaluza: ideas, } \\
\text { personas e instrumentos para dirigir la } \\
\text { empresa }\end{array}$ & Harvard Deusto Márketing y Ventas \\
\hline Alkaid: revista multitemática & HSM Management \\
\hline Anuario de Derecho Municipal & INGE CUC \\
\hline Arquitectura Viva & Ipmark: Información de publicidad y marketing \\
\hline Arte, individuo y sociedad & Mundo electrónico \\
\hline Automática e instrumentación & Papers: Regió Metropolitana de Barcelona \\
\hline $\begin{array}{c}\text { Azarbe: Revista Internacional de Trabajo } \\
\text { Social y Bienestar }\end{array}$ & $\begin{array}{c}\text { PARJAP: Boletín de la Asociación Española de } \\
\text { Parques y Jardines }\end{array}$ \\
\hline Bit & Parques y jardines \\
\hline Byte España & Personal Computer \& Internet \\
\hline Cambio 16 & $\begin{array}{c}\text { Perspectiva CDTI: revista de innovación } \\
\text { tecnológica }\end{array}$ \\
\hline $\begin{array}{c}\text { Carreteras: Revista técnica de la Asociación } \\
\text { Española de la Carretera }\end{array}$ & Política exterior \\
\hline $\begin{array}{c}\begin{array}{c}\text { Cercha: revista de los aparejadores y } \\
\text { arquitectos técnicos }\end{array} \\
\end{array}$ & $\begin{array}{c}\text { Práctica urbanística: Revista mensual de } \\
\text { urbanismo }\end{array}$ \\
\hline $\begin{array}{c}\text { CIC: publicación mensual sobre arquitectura } \\
\text { y construcción }\end{array}$ & $\begin{array}{l}\text { Qualitas Hodie: Excelencia, desarrollo } \\
\text { sostenible e innovación }\end{array}$ \\
\hline $\begin{array}{l}\text { Consultor de los ayuntamientos y de los } \\
\text { juzgados: Revista técnica especializada en } \\
\text { administración local y justicia municipal }\end{array}$ & Retema: Revista técnica de medio ambiente \\
\hline Dínamo técnica: revista gallega de energía & Revista AHCIET: revista de telecomunicaciones \\
\hline Dossier Europa & $\begin{array}{c}\text { Revista APD: Asociación para el Progreso de la } \\
\text { Dirección }\end{array}$ \\
\hline Dyna & $\begin{array}{c}\text { Revista Aranzadi de derecho y nuevas } \\
\text { tecnologías }\end{array}$ \\
\hline Ecología política & Revista de estudios locales "Cunal" \\
\hline Economía aragonesa & $\begin{array}{l}\text { Revista de Obras Públicas: Órgano profesional } \\
\text { de los ingenieros de caminos, canales y puertos }\end{array}$ \\
\hline El Instalador & Revista Gestão \& Conexões \\
\hline El profesional de la información & $\begin{array}{c}\text { Revista Internacional de Protocolo: Ceremonial, } \\
\text { Etiqueta, Heráldica, Nobiliaria y Vexilogía }\end{array}$ \\
\hline Electra & Seguritecnia \\
\hline Electrónica y comunicaciones & Sesenta y más \\
\hline $\begin{array}{c}\text { Emprendedores: las claves de la economía y } \\
\text { el éxito profesional }\end{array}$ & Sociedad y utopía: Revista de ciencias sociales \\
\hline Energía de hoy.com & $\begin{array}{c}\text { Tecnohotel: revista profesional para la } \\
\text { hostelería y restauración }\end{array}$ \\
\hline Equipamiento y servicios municipales & Temas para el debate \\
\hline $\begin{array}{l}\text { Eurofach electrónica: Actualidad y } \\
\text { tecnología de la industria electrónica }\end{array}$ & $\begin{array}{l}\text { UOC Papers: revista sobre la sociedad del } \\
\text { conocimiento }\end{array}$ \\
\hline
\end{tabular}

Tabla 1.- Listado de las 58 publicaciones periódicas que albergan artículos sobre smart cities en lengua española o lenguas cooficiales. Elaboración propia a partir de los datos de Dialnet e ISOC. 
A la hora de subrayar cuáles son las revistas más productivas y volcadas en el área de las smart cities, destacan las siguientes publicaciones, por número de trabajos publicados en la materia, lo que las convierten en publicaciones de referencia. Sin duda alguna, la revista de referencia es la Revista de Obras Públicas, que es el órgano oficial de los ingenieros de caminos, canales y puertos, con un total de 14 artículos (11,6 por ciento de las unidades analizadas). Le sigue la revista Equipamientos y Servicios Municipales, con un total de 11 trabajos publicados (9,16 por ciento), la revista periodística Cambio 16, con 8 artículos (6,66 por ciento). Con 4 trabajos (3,33 por ciento) están las revistas Bit, la publicación Carreteras (que es la revista técnica de la Asociación Española de la Carretera) y, finalmente, la revista Electra. Con 3 artículos (2,5 por ciento) están las revistas Automática e Instrumentación y la publicación Electrónica y Comunicaciones.

\begin{tabular}{|c|c|}
\hline \multicolumn{2}{|c|}{ Autores especialistas en el campo de las smart-cities } \\
\hline Abella García, Alberto & Morales Carballo, Luis \\
\hline Aguado Molina, Jesús (coord.) & Morata Morata, Antonio \\
\hline Agudo Martínez, María Josefa & Moreno Cegarra, José Luis \\
\hline Albertos Puebla, Juan Miguel (coord.) & Moreno, Luis \\
\hline Alcázar, Pilar & Muñoz De Dios, María Dolores \\
\hline Aldama Gutiérrez, Eneko Aritza & Muñoz Gutiérrez, Luis \\
\hline Alfaro Martínez, Elena & Navarro Gómez, Carmen \\
\hline Alfonso Avello, Antonio & Navarro Ruiz, Manuel \\
\hline Álvarez Fuente, José Ángel & Navarro Vera, José Ramón (coord.) \\
\hline Álvarez Vázquez, Luis (coord.) & Navío Marco, Julio \\
\hline Anaya Díaz, Jesús & Negreira Souto, Carlos \\
\hline Arnal,José Carlos & Núñez Freile, José Luis \\
\hline Ausaverri, Manuel & Ojeda Rivera, Juan Francisco \\
\hline Aymerich, M. & Ondategui Rubio, Julio César \\
\hline Balaguer,Bernardo & $\begin{array}{c}\text { Ortega Ramírez, Juan Antonio (coord. y } \\
\text { aut.) }\end{array}$ \\
\hline Bengochea Martínez, Luis (coord.) & Ortega, Ángel \\
\hline Bizelli, José Luis (ed.lit.) & Ortiz de Urbina Criado, Marta \\
\hline Blanco Silva, Fernando (coord.) & Pablos Heredero, Carmen de \\
\hline Bonet Sánchez, Eduardo & Parajó Calvo, Bernardo (coord.) \\
\hline Buendía Iglesias, Ricardo & Pascual Ruiz-Valdepeñas, Henar (coord.) \\
\hline Cadena León,Adriana María & Pascual Vives, Francisco José (coord.) \\
\hline Cama Pinto, Alejandro & Pedreño Muñoz, Andrés \\
\hline Cama Pinto, Dora & Pellejero, Gonzalo \\
\hline
\end{tabular}




\begin{tabular}{|c|c|}
\hline Campos López, Juan José & Peña de Carrillo, Clara Inés \\
\hline Casado,Héctor & Perales, Marta de \\
\hline Chaparro Mantilla, Mónica Liliana & Pérez, Carol \\
\hline Chulilla Cano, Juan Luis & Pérez Candela, Alejandro \\
\hline Conti, Alfredo (coord.) & Pernías, Pedro \\
\hline Davara Rodríguez, Miguel Ángel & $\mathrm{Pi}$, Xavier \\
\hline De la Hoz Franco, Emiro & Pires, António A. \\
\hline Fernández, Eduardo & Plaza Penadés, Javier \\
\hline Fernández, Manu & Portillo Navarro, María José (coord.) \\
\hline Fernández-Montes González, Alejandro & Rabanal, Ch. \\
\hline Franco, Ainhoa & Ramírez Autrán, Rodrigo \\
\hline Fuente Robles, Yolanda María de la & Ramón, Ana \\
\hline Galache López, José Antonio & Rayón Martín, Fernando \\
\hline García García, Antonio (coord. y autor) & Ribera Fumaz, Ramón \\
\hline García López, Paloma & Rivas Pereda, Carlos \\
\hline García Rubio, Fernando & Rodríguez Bustamante, Pablo \\
\hline García, Javier & Rodríguez Gamo, José Luis \\
\hline Ghilès, Francis & Ros, Germán (coord.) \\
\hline $\begin{array}{l}\text { González Fernández, Roberto Carlos } \\
\text { (coord.) }\end{array}$ & Ruiz, Miguel \\
\hline González Torroba, Pedro & Saavedra, Constanza \\
\hline Guerrero Ortega, Antonio (coord.) & Sala, Jordi \\
\hline Hernández Galán, Jesús & Salamanca, Rafael \\
\hline Hidalgo, Miguel Ángel (coord.) & Sánchez Hernández, José Luis (coord.) \\
\hline Izquierdo Vilavella, Xavier & Sánchez Venzalá, José Ignacio \\
\hline Jabonero Blanco, Mariano (ed.lit.) & Sancho Royo, Fernando \\
\hline Lindsay, Greg & Sarmiento Díez, Oriol (coord.) \\
\hline Lissavetzky Díez, Jaime & Sartor, Michael \\
\hline Löffler, Peter & Seepol, Ralf (coord.) \\
\hline López, Juan Carlos & Seisdedos Castilla, Soledad \\
\hline Losada Pereda, María Begoña & Seisdedos, Gildo \\
\hline Losada Suárez, Santiago & Serna Hernáiz, Iñigo de la \\
\hline March Corbella, Hugo & Soria Rodríguez, Marcelo \\
\hline Marco Such, Manuel & Tejedor Martínez, Cristina (coord.) \\
\hline Marín, Jordi & Torres Gutiérrez, Francisco José \\
\hline Martín Bris, Mario (ed.lit.) & Trachana, Angelique \\
\hline Martín García, Jorge & Trujillo Lora, Juan Carlos \\
\hline Martínez Arias, Adriana (ed.lit.) & Valdenebro García, José Vicente \\
\hline Martínez Díaz, María del Mar & Vilatersana, Climent \\
\hline Martínez Madrid, Natividad (coord.) & Villa, Juan Manuel \\
\hline Méndez Gutiérrez del Valle, Ricardo (coord.) & Villafáfila, Roberto \\
\hline Mercader-Moyano, Pilar & Villanueva, Félix Jesús \\
\hline Merino Estrada, Valentín & Vives Riera, Antoni \\
\hline
\end{tabular}




\begin{tabular}{|c|c|}
\hline Millán Jiménez, Ana María (coord.) & Woodley, Tedy \\
\hline Mitchell, William J. & Zubieta, Roberto \\
\hline
\end{tabular}

Tabla 2.- Listado de los 134 autores de las 120 unidades de análisis sobre smart cities en lengua española o lenguas cooficiales. Elaboración propia a partir de los datos de Dialnet e ISOC.

Respecto a la filiación de los autores destacan la Universidad de Talca (Chile) con ocho aportaciones o la de Alcalá de Henares (España) con tres trabajos y la Universidad Complutense de Madrid (España) con otras tres aportaciones. Otras instituciones españolas de investigación cuyos autores han publicado con proyectos ligados al tema son la Universidad Autónoma de Madrid, la Universidad Pontificia de Salamanca, la Universidad de Huelva y el Consejo Superior de Investigaciones Científicas (CSIC). Cabe destacar un asunto importante, aunque la producción analizada es en lengua española (y cooficiales) de países como Chile, España o Argentina, hay autores hispanohablantes ligados también a instituciones, universidades o centros de investigación internacionales de países como Reino Unido, Italia, Holanda, Estados Unidos y Alemania. Se trata en la mayoría de los casos de autores hispanohablantes que desarrollan su carrera profesional o investigadora en centros de estos países de Europa o América. De todos los trabajos analizados, solo se han encontrado dos trabajos en otras lenguas cooficiales del Estado Español diferentes al castellano, y se trata de dos trabajos en catalán (en Papers: Regió Metropolitana de Barcelona). No se han localizado ni por tanto analizado trabajos en gallego o euskera. Hay un artículo en español en la Revista Gestão \& Conexões y también publica en castellano otro trabajo la publicación Dínamo técnica: revista gallega de energía.

En relación a la naturaleza de las publicaciones, hay que destacar que se produce una gran desproporción a favor de los artículos especializados (62 por ciento del total de trabajos analizados), frente a los capítulos de libros. Solo hay cinco capítulos de libros y dos libros completos. En relación a las revistas, es imposible delimitar una línea clara y concisa entre las publicaciones altamente especializadas, especializadas y de divulgación. Sin embargo, sí es posible diferenciar medios generalistas de información (como es por ejemplo el caso de la revista Cambio 16). Hay publicaciones híbridas difíciles de clasificar en una de las anteriores categorías. 
Respecto a la temática de las publicaciones o áreas de especialización desde las que los autores lanzan sus estudios y propuestas, el abanico cubre campos del conocimiento como la Arquitectura y Urbanismo (a través de publicaciones como Arquitectura Viva; Cercha: revista técnica de los aparejadores y arquitectos técnicos; Hábitat y Sociedad), las Ciencias Políticas (Temas para el Debate, Sociedad y Utopía, Política Exterior), el mundo del Derecho (Anuario de Derecho Municipal; Consultor de los ayuntamientos y de los juzgados: Revista técnica especializada en administración local y justicia municipal; Revista Aranzadi de derecho y nuevas tecnologías) o las Ciencias Económicas y Empresariales (Harvard Deusto Márketing y Ventas, Ipmark: Información de publicidad y marketing).

Respecto al perfil de los autores, es preciso destacar la pluralidad de perfiles. Destacan autores ligados al mundo de la política. Así entre los textos analizados, se encuentran trabajos de destacados líderes políticos de diferente signo político. Es el caso de Jaime Lissavetzky Díez (Partido Socialista Obrero Español) o de Iñigo de la Serna Hernáiz (Partido Popular). Lissavetzky ha sido Secretario de Estado para el Deporte del Gobierno de España, consejero autonómico del Gobierno de la Comunidad de Madrid, parlamentario en la Asamblea de Madrid y concejal del Ayuntamiento de Madrid. Por su parte, De la Serna ha sido alcalde de Santander y presidente de la Federación Española de Municipios y Provincias, y en su calidad de ingeniero de caminos, canales y puertos, es también experto en el tema de las ciudades inteligentes.

Sin duda alguna, una vez analizado el contenido de las 120 unidades de análisis, destaca el peso de las entidades políticas, sobre todo las locales. Como demuestra el análisis de contenido, los ayuntamientos, al ir avanzando en este proceso inteligente de modernización, han valorado la oportunidad de establecer estrategias de colaboración y coordinación para aunar esfuerzos. Conceptos como sostenibilidad, movilidad o destino inteligente han permitido integrar diferentes ayuntamientos bajo un mismo proyecto. Una de las organizaciones surgida de esta confluencia es la Red Española de Ciudades Inteligentes (RECl), que ya cuenta con más de 60 ciudades. Cabe destacar el esfuerzo que empresas y ayuntamientos están desarrollando para definir estándares e indicadores en el seno del Grupo Técnico de Normalización 178 de la Asociación Española de Normalización y Certificación (AENOR), en cuya revista se han publicado en el período 
analizado textos sobre smart cities.

Del análisis del estado de la cuestión también destacan en nuestro país otras iniciativas como la Alianza Inercia, una alianza multisectorial coordinada por la Comisión de Smart Cities de la Asociación de Empresas de Electrónica (AMETIC), y la plataforma tecnológica Es.Internet, han constituido grupos para debatir y acordar estrategias que acercan las capacidades empresariales a las necesidades de las ciudades.

Otra iniciativa descubierta tras el análisis de información es el proyecto IntelligenTIC, de la Confederación Española de Empresas de Tecnologías de la Información, Comunicación y Electrónica (CONETIC), que promueve la integración de soluciones implantadas por diferentes pequeñas y medianas empresas (pymes), con el fin de reforzar las competencias empresariales individuales con productos y servicios interoperables, que incrementan el retorno de la inversión de las ciudades. Finalmente en el caso de la bibliografía sobre smart cities hay que destacar los trabajos que son frutos de congresos y eventos que cada año se celebran en nuestro país. Destacan las publicaciones ligadas al Congreso Internacional Ciudades Creativas, celebrado en 2015 en Santiago de Compostela. Y, del mismo modo, es particularmente reseñable el trabajo y la visibilidad internacional alcanzados por nuestro país gracias a la Fundación Mobile World Capital de Barcelona, un activo a preservar.

De la misma manera, esta obligación de velar por el futuro de las ciudades inteligentes con el fin de mejorar la calidad de vida de todos es responsabilidad de todos, incluyendo también las Ciencias de la Información y de la Comunicación, con especial atención a la Biblioteconomía y Documentación, pues, como afirma Anglada (2014: 603), si la generación actual de bibliotecarios no es capaz de introducir cambios radicales en el papel de las bibliotecas, su continuidad está seriamente amenazada.

\section{Conclusiones}

El fenómeno de las ciudades inteligentes (o smart cities) es ya una realidad. El estudio de la producción bibliográfica demuestra que la comunidad hispanoparlante utiliza 
indistintamente tanto el término propio en español como el original en inglés, motivo por el que este trabajo también ha usado las dos fórmulas posibles reconocidas por la comunidad académica. No es un proyecto de ciencia ficción ni un futurible lejano. Es todo un reto para administradores, políticos, ingenieros, y también para la comunidad académica y científica, no sólo para los profesionales.

Los administradores públicos y gestores políticos son los responsables oficiales del cuidado y mejora de nuestros entornos urbanos, pero en la práctica no son los únicos que deben llevar a cabo esta tarea. No deben estar solos a la hora de afrontar problemas de organización social, estructura urbana o impacto ambiental. La comunidad científica y académica, por tanto los miembros de la universidad, también tenemos nuestra responsabilidad. No es una responsabilidad que nos viene dada por nuestra posición como consumidores de recursos materiales y energéticos, o fuente de emisión de residuos y contaminantes. Es nuestra misión científica y cívica la que tiene también como fin servir al interés general y al bien común.

Desde la literatura científica y profesional centrada en el estudio de las ciudades inteligentes, se ve desde el principio una clara conciencia social que tiene como fin último el intentar mejorar la relación con el entorno, mantener la calidad y el coste de los servicios prestados o la capacidad para reorganizar sus estructuras, dando respuesta a nuevas necesidades, especialmente en los textos escritos durante los últimos años de la crisis económica y social que ha azotado España.

La literatura dedicada a la historia de las smart cities en nuestro país establece que el nacimiento de las primeras investigaciones y publicaciones del siglo XXI tenían una honda impronta marcada por los patrones de investigación estadounidenses. Pese a ello, una de las primeras conclusiones es que, incluso a día de hoy, no hay consenso sobre el origen de la disciplina en España y a este respecto existen diferentes corrientes, puesto que hay trabajos del área de la Arquitectura, las Ciencias Políticas y Jurídicas, o las Ciencias de la Comunicación y las Nuevas Tecnologías, y aunque el sello anglosajón es obvio, las fuentes y referentes utilizadas corresponden a áreas del conocimiento muy diferentes entre sí. 
Frente a esa compleja realidad académica, la realidad política en el caso de España es que un número significativo de ciudades de todo el país han ido poco a poco adoptando las nuevas tecnologías para realizar una gestión más eficiente en la prestación de sus servicios públicos, la redefinición de los mismos o el replanteamiento de las relaciones con ciudadanos, turistas, empresas y proveedores. Esta realidad no es un resultado aislado. Sin duda es fruto de la cooperación entre ayuntamientos, universidades, thinktanks, grupos de investigación y el sector privado. No debe por tanto aislarse o apartarse la investigación universitaria en materia de ciudades inteligentes de la puesta en marcha de programas reales en las ciudades de nuestro país. A esta realidad ha contribuido también mucho la tan criticada en otras ocasiones existencia o presencia de universidades repartidas por toda la geografía española.

La investigación sobre smart cities es necesaria y es un campo en expansión. Tiene ya bastante dicho y publicado, como se ha demostrado en el análisis, pero la mayor parte está por ser escrito. En un breve espacio de tiempo, gracias al desarrollo tecnológico de nuestras ciudades, tendremos incluso otra forma de viajar o realizar actividades en nuestro día a día urbano. De hecho, el tratamiento de la big data cambiará también nuestros hábitos de consumo, o mejor dicho, hará que los productos y marcas se adapten más a nuestras necesidades. También cambiará la forma en la que se aprende y estudia Así, dentro de poco los que son hoy alumnos de educación infantil de cualquier centro de España, probablemente se especializarán en trabajos que todavía no existen y utilizarán a diario una tecnología que a día de hoy aún no ha sido inventada.

Este desarrollo tecnológico, base de las smart cities, que ya vemos en nuestras vidas desde que usamos un parquímetro municipal hasta en el momento de pedir una cita con la administración pública, conllevará que las ciudades españolas tengan que estar preparadas en los próximos años para prestar servicios, fundamentalmente no administrativos, de manera sostenible, digital, rápida y ágil.

En relación al análisis de las 120 unidades tratadas es preciso insistir al respecto que a pesar de encontrar diferentes posturas respecto a la evolución y la relación de los 
términos es, hoy por hoy, innegable la vinculación entre las diferentes concepciones del fenómeno de las smart cities o ciudades inteligentes en el contexto académico español, independientemente de que la temática o aproximación al objeto de estudio se produjera desde la Arquitectura, Ingeniería, Economía, Ciencias Jurídicas o de la Comunicación. Así, unidades localizadas en las búsquedas de "smart city" o "ciudad inteligente", tanto en plural como en singular, tiene siempre en común un fenómeno u objeto de estudio que a veces es difícil de definir, pero que siempre tiene algo en común con otros textos independientemente del tipo de publicación o el origen del autor, que como hemos visto puede ser de uno u otro lado del Atlántico y de formaciones o áreas de conocimiento muy diferentes. Atender a las necesidades de agua potable, transporte, aire limpio, movilidad, tecnología accesible, información, bienestar y calidad de vida de su habitantes es un reto de extraordinaria magnitud y una oportunidad para la sociedad que despierta el interés de diferentes autores desde diversas áreas de conocimiento o especialización, lo que hace del fenómeno de las smart cities un objeto de estudio plural, una disciplina transversal y una gran oportunidad para todos, gestores e investigadores, que tendrán durante los próximos años el reto de trabajar codo con codo.

\section{Referencias}

Agenda Digital del Gobierno de España (2015). http://www.agendadigital.gob.es/Paginas/Index.aspx

Asociación Española de Normalización y Certificación (AENOR) (2015).

Plan Nacional de Ciudades Inteligentes - Grupo Técnico de Normalización 178 de AENOR (AEN/CTN 178/SC2/GT1 N $003 . \quad$ http://www.agendadigital.gob.es/planesactuaciones/Bibliotecaciudadesinteligentes/Material \%20complementario/normas_ciudades_inteligentes.pdf

Anglada, Lluís (2014). Are libraries sustainable in a world of free, networked, digital information? // El Profesional de la Información. ISSN 1386-6710/ISSN-e 1699-2407. 23:6 (Noviembre-diciembre de 2014) 603-611. http://dx.doi.org/10.3145/epi.2014.nov.04 
Cabezuelo Lorenzo, Francisco (2013). Cinco años de crisis en el mercado de la comunicación (2008-2013) // Historia y Comunicación Social. ISSN 1137-0734. 18: Extra 3 (Diciembre) 703-715.

http://dx.doi.org/10.5209/rev_HICS.2013.v18.44358

Caldevilla Domínguez, David (2010). Internet como fuente de información para el alumnado universitario. // Cuadernos de Documentación Multimedia. ISSN-e 1575-9733. Núm. 21, 141-157.

Castells, Manuel (2008). Comunicación, poder y contrapoder en la sociedad red (I). Los medios y la política. // TELOS: Cuadernos de comunicación e innovación. ISSN 0213-084X. $74,13-24$.

Llobell, Juan (2012). Ciudades inteligentes, un planeta de urbanitas. Revista Capital. http://www.capital.es/2012/04/13/ciudades-inteligentes-un-planeta-de-urbanitas/

Marcos Recio, Juan Carlos, Sánchez Vigil, Juan Miguel \& Serrado Gutiérrez, María (2009). Nuevos paradigmas periodísticos y documentales en los periódicos digitales: estudio de casos en España. // Investigación Bibliotecológica. ISSN: 0187-358X. 23: 49 (Septiembrediciembre) 43-65.

Pascual, Rafael (2012). Dossier Smart Cities de Revista Capital. Disponible en: http://www.indracompany.com/sites/default/files/indra_76-84smartcities.compressed.pdf

Sánchez Martínez, María \& Ibar Alonso, Raquel (2015). Convergence and interaction in the new media: typologies of prosumers among university students. // Communication \& Society. ISSN-e: 2174-089528(2), 87-99

Verbeke, Mathias et al. (2014). When two disciplines meet, Data Mining for Communication Science. En $64^{\text {th }}$ Annual Meeting of International Communication 
Association (ICA) Conference. Seattle, Estados Unidos. https://lirias.kuleuven.be/handle/123456789/436424 